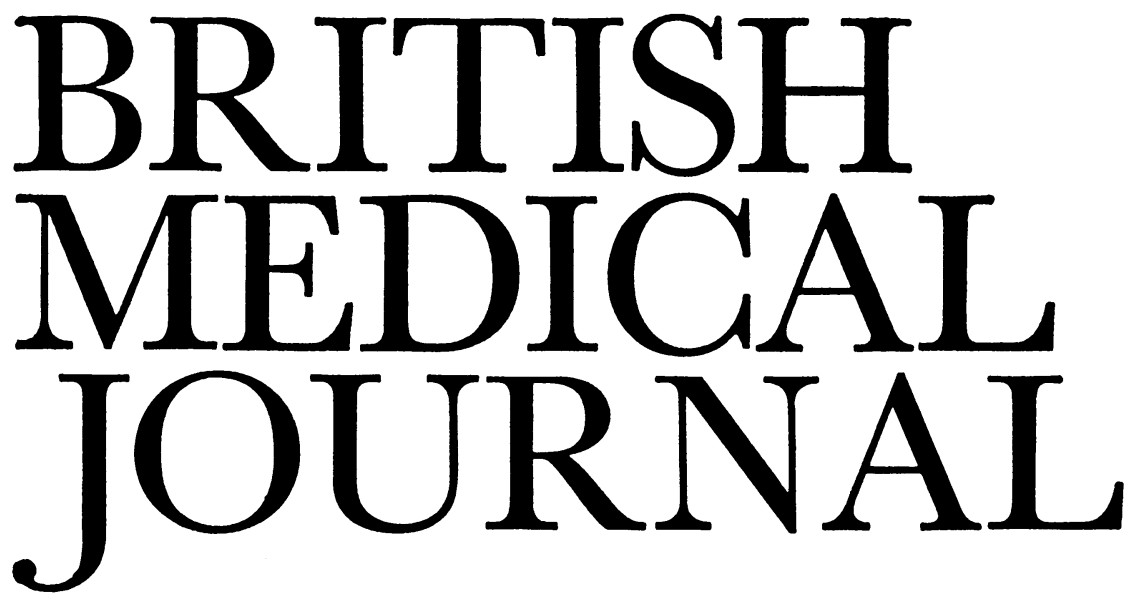

LONDON, SATURDAY 17 JUNE 1978

\title{
Nutrition and the brain
}

Whether or not malnutrition may reduce an individual's capacity to realise his full intellectual potential by affecting the physical structure of his brain is not a new question. In the past 125 years it has several times seemingly been laid to rest by the invulnerability of the adult brain to the most severe, even fatal, starvation. More recently, however, animal experiments have shown the brain's surprising sensitivity to quite moderate nutritional growth restriction, so that interest has been reawakened; and the last decade has seen an enormous amount of both laboratory and epidemiological investigation. ${ }^{12}$

Two major obstacles impede any clear solution, and these are likely to remain. One is our virtually complete ignorance of the physical basis of higher mental function. The other is the apparently hopeless tangle of interdependent environmental influences on the shaping of personality and intellect, ${ }^{3}$ from which it may never be possible to distinguish malnutrition (or any other single factor) as a specific cause of observed deficit. ${ }^{4}$ Variations in social class, for example, provide a galaxy of factors, to which nutrition makes only a single contribution. Couple with these difficulties the naive but common belief that intellect can be defined and in some way measured, and the scene is set for the topic to be abandoned once again by serious investigators, this time because of the daunting complexity of avoiding the pitfalls created by this belief.

Nor are these the only impediments to progress. The inevitable political interest in a topic with such pressing social implications has made it easy for investigators in almost any discipline to obtain support for research into any aspect of nutrition and the brain-often without the necessary humility. One disturbing feature has been the way the expression "mental retardation" has been linked-quite unscrupulously -with malnutrition, the literal meaning of the term being exploited to suggest that a severe degree of brain damage may in some way be caused by malnutrition. Serious as the effects of poor nutrition on the developing brain may well be, they are already seen to stop far short of mental retardation in its usual sense.

Amid all the confusion one conclusion has emerged from the recent experimental work: there are clearly periods during its growth and development when the brain is vulnerable. ${ }^{25}$ Brain growth may be impaired in such a way that, while there has been no destruction or lesions, adequate numbers (and even sizes) of components have failed to assemble; thus the mature product is not only deficient but, in a diffuse, quantitative sense, distorted. Quite moderate nutritional restriction of growth during the period when important structures normally appear may impede their development in the usual quantities, and some of the resulting histological imbalance is resistant to rehabilitation by restoring an adequate diet. Permanent effects are also found in some of the brain's metabolic and biochemical functions, certain of which (such as catecholamine metabolism ${ }^{6}$ ) are possibly related to higher mental function. Not only can inadequate nutrition make the brain permanently somewhat smaller than normal (possibly of no greater significance than the fact that most women have smaller brains than men), but the smallness is also not uniform. The cerebellum, for example, is selectively affected and within the cerebellum only certain structures are affected ${ }^{6}$; in animal studies this is associated with detectable though minimal clumsiness. ${ }^{2}$ In an animal given a restricted diet during the vulnerable period there are substantially fewer synapses per neurone in the cerebral cortex ( Cragg $^{7}$ claims a deficit of 30-40\%), though whether such a structural distortion is permanent is not yet known. New quantitative histological techniques such as stereology and automatic counting have scarcely been applied to the brain, and would almost certainly be productive.

Inevitably, these findings come from studies in animals. Whether they have meaning for human neuroanatomy remains an open question; if they have, the supreme question-whether they matter at all to intellectual function-will probably never be answered with scientific finality. $\mathrm{Cheek}^{8}$ has claimed that results of this kind derived from small laboratory animals cannot be replicated in subhuman primates and hence are not relevant to man. Dobbing, ${ }^{9}$ however, has argued that such extrapolations between species have not been fairly made: they have not taken enough account either of the obvious differences between species in the timing of the developmental vulnerable period in relation to birth or of its different duration in fast and slowly growing species. Dobbing claims that the period of growth of the human brain corresponding to the vulnerable period in animals extends from about midgestation at least until the second birthday. All these months should be seen as a period both of vulnerability and of opportunity to promote good brain growth. The earlier ${ }^{10}$ as well as the continuing studies of Cravioto and others ${ }^{11} 12$ in underprivileged communities do seem to support this view, though there are many examples of extreme deprivation during this period that have not resulted in measurable intellectual deficit. ${ }^{13}$

Perhaps as humans we have a great capacity to compensate 
for the deleterious effects of such adversity, provided that it is compensated sufficiently by all other aspects of the environment-as well as the stimulus that used to be called tender, loving care. Perhaps malnutrition reduces human achievement only when it is added to other adversity. But, as we must confess to our collective shame, it usually is.

${ }^{1}$ Dodge, P R, Prensky, A L, and Feigin, R D, Nutrition and the Developing Nervous System. St Louis, Mosby, 1975.

2 Dobbing, J, in Scientific Foundations of Paediatrics, eds J A Davis and J Dobbing. London, Heinemann, 1974.

${ }^{3}$ Pollitt, E, and Thomson, C, in Nutrition and the Brain, vol 2, eds R J Wurtman and J J Wurtman. New York, Raven, 1977.

${ }^{4}$ Cravioto, J, et al, Acta Paediatrica Scandinavica, 1967, 56, 71.

${ }^{5}$ Davison, A N, British fournal of Psychiatry, 1977, 131, 565.

${ }^{6}$ Smart, J L, et al, Paediatric Research, 1976, 10, 807.

${ }^{7}$ Cragg, B G, Brain, 1972, 95, 143.

${ }^{8}$ Cheek, D B (ed), Fetal and Postnatal Cellular Growth. London, John Wiley, 1975.

${ }^{9}$ Dobbing, J, Nutrition Reports International, 1973, 7, 401.

${ }^{10}$ Cravioto, J, and Robles, B, American Journal of Orthopsychiatry, 1965, 35, 449.

${ }^{11}$ Hertzig, M E, et al, Pediatrics, 1972, 49, 814.

12 Birch, H G, and Richardson, S A, in Nutrition, the Nervous System, and Behavior. Washington, Pan American Health Organisation, 1972.

13 Lloyd-Still, J D (ed), Malnutrition and Intellectual Development. Lancaster, Medical and Technical Publishing, 1976.

\section{Massive haemoptysis}

All too often massive haemoptysis is a medical catastrophe: patients may die within a few minutes from asphyxiation before conservative or surgical measures can be carried out. Among the causes of massive haemoptysis are mycetoma, bronchial carcinoma, chronic cavitated pulmonary tuberculosis, lung abscess, and bronchiectasis; it has also been reported in necrotising arteritis. We should not forget, however, that pulmonary tuberculosis in its acute stageswith little or no obvious cavitation-may cause massive fatal pulmonary bleeding. A recent report described five deaths from haemoptysis due to acute pulmonary tuberculosis in a single hospital within six years. ${ }^{1}$ All the patients were aged 45 or less, and, though all had been noted to have haemoptysis on admission, none had appeared to have such destructive disease that a massive haemoptysis seemed likely. Tuberculous cavities have a particularly rich bronchial blood supply, and when heavy bleeding occurs it comes from dilated bronchial arterioles $^{2}$; such dilated bronchial vessels are also found in mycetoma and bronchiectasis.

How should the clinician attempt to predict the outcome in severe haemoptysis? The only reliable guide is that the faster the rate of blood loss the more likely is the patient to die, the mortality rate being about $75 \%$ if blood loss exceeds $600 \mathrm{ml}$ in six hours. ${ }^{3}$ Surgical intervention is not an easy solution, however, even when technically practicable: the mortality rate is between $15 \%$ and $20 \%$ after resection of bleeding lesions. ${ }^{5-7}$ Surgical treatment is often precluded by the poor respiratory reserve often found in patients with mycetoma and widespread pulmonary tuberculosis or by inoperable or disseminated carcinoma. An alternative may be emergency induction of an artificial pneumothorax. ${ }^{8}$

The patient who develops more than minor haemoptysis should rest in bed-lying on the affected side if this is known. A surgeon should assess his condition early on. The pulse and blood pressure should be monitored as well as the amount of blood lost-in view of the prognostic importance of the rate of haemorrhage. Careful radiographic assessment, including tomography if necessary, is important. Six units of blood should be made available and the patient must clear the airways by gentle coughing; he should not be sedated.

Bronchoscopy is central to management. " Using the rigid $\stackrel{\text { W }}{7}$ instrument, the airways can be cleared of blood, some of it in the form of clots. There is a common misconception that when the patient is bleeding bronchoscopy is valueless for identifying the source precisely. In one series, however, it identified the $\stackrel{9}{\stackrel{9}{9}}$ site of bleeding in 65 out of 67 patients, ${ }^{3}$ and in another in 18 을 out of 21 patients. ${ }^{9}$ Occluding the bronchial artery by embolisa- $\frac{\bar{\sigma}}{\overline{0}}$ tion is a skill possessed by few clinicians, but many more are $\frac{\sigma}{\sigma}$ skilled in the use of the fibreoptic bronchoscope, so that the $\triangle$ method of arresting bleeding described by Saw et $a l^{4}$ is important. Combining flexible fibreoptic bronchoscopy with $\overrightarrow{0}$ systematic lavage of the tracheobronchial tree they identified $\overrightarrow{\vec{H}}$ the source of bleeding and stopped it in all their 10 patients $\omega_{0}$ (six of whom had pulmonary tuberculosis). The bleeding was halted by balloon tamponade of the affected segment with a Fogarty catheter; the balloon was deflated after 24 hours and $\dot{\sigma}$ the catheter removed a few hours later.

Clearly, then, aggressive methods of diagnosis permit early surgical intervention should haemoptysis increase. Oु Nevertheless, the selection of patients for intervention remains 윽 a daunting problem.

${ }^{1}$ Middleton, J R, et al, Chest, 1977, 72, 601.

2 Cudkowicz, L, Thorax, 1952, 7, 270.

3 Crocco, J A, et al, Archives of Internal Medicine, 1968, 121, 495.

${ }^{4}$ Saw, E, et al, Chest, 1976, 70,589.

5 Gourin, A, and Garzon, A A, Annals of Thoracic Surgery, 1974, 18, 52.

${ }^{6}$ Mattox, K L, and Guinn, G A, Annals of Thoracic Surgery, 1974, 17, 377.

${ }^{7}$ McCollum, W B, et al, Chest, 1975, 67, 152.

${ }^{8}$ Jenkins, D E, and Wolinsky, E, in Textbook of Pulmonary Diseases, 2nd edn, ed G L Baum, p 331. Boston, Little and Brown, 1974.

${ }^{9}$ Pursel, S E, and Lindskog, G E, American Review of Respiratory Diseases, $1961,84,329$.

\section{Hypertension and oral contraceptives}

Practically all women who take oestrogen-containing oral contraceptives have a rise in blood pressure. Severe or even $ᄋ$ malignant hypertension is rare, but about $4 \%$ develop $N$ diastolic blood pressures of $90 \mathrm{~mm} \mathrm{Hg}$ or more. ${ }^{2}$ This preva- $\rightarrow$ lence is about twice that in women of the same age not using oral contraceptives. Furthermore, there is a significant and $N$ sustained rise in blood pressure even in women who do not develop hypertension. A major prospective study in Glasgow showed a mean increase of $9.2 \mathrm{~mm} \mathrm{Hg}$ in systolic pressure and 0 $5.0 \mathrm{~mm} \mathrm{Hg}$ in diastolic pressure ${ }^{3}$ among 83 women, though in none did the diastolic pressure exceed $90 \mathrm{~mm} \mathrm{Hg}$.

The long-term effects of this change in blood pressure are unknown. Many epidemiological studies of large populations have shown that the height of the systolic blood pressure is a $\frac{O}{\mathbb{D}}$ potent predictor of subsequent cardiovascular disease. ${ }^{4}$ Small rises which remain within the range conventionally regarded as normal or clinically insignificant might therefore be $\frac{8}{0}$ expected to be associated with increased risk-and, indeed, an excess of deaths from non-rheumatic heart disease and cerebrovascular disease is found particularly in older women taking oral contraceptives, though in such studies antecedent hypertension has not been documented. ${ }^{5-7}$

The results of attempts to understand the mechanism of the 